\title{
Improvements encoding energy benefit in protected telecommunication data transmission channels
}

\author{
Juliy Mikolayovych Boiko, ${ }^{1,}$, Alexander Ivanovych Eromenko \\ ${ }^{1}$ Department of Radio Engineering and Communication, Khmelnitsky National University, Khmelnitsky, Ukraine \\ ${ }^{2}$ Department of Physics and Electrical Engineering, Khmelnitsky National University, Khmelnitsky, Ukraine
}

Email address:

boiko_julius@ukr.net (J. M. Boiko), sashaf52@mail.ru (A. I. Eromenko)

To cite this article:

Juliy Mikolayovych Boiko, Alexander Ivanovych Eromenko. Improvements Encoding Energy Benefit in Protected Telecommunication Data Transmission Channels. Communications. Vol. 2, No. 1, 2014, pp. 7-14. doi: 10.11648/j.com.20140201.12

\begin{abstract}
The features of using noise resistant encoding for protection from errors in digital data transmission channels with phase modulation have been analyzed. The building principles and encoder and decoder schemes of turbo-code were observed. The digital data transmission system with outer and inner turbo-encoder has been developed. The recommendations to type and structure of cascade encoder components have been given. Analytical relationships and research results describing bit error probability of cascade turbo-encoder components are represented. By using simulative modeling tools Matlab-Simulink the basic characteristics and features of digital transmission channel with cascade turbo-encoding and digital phase modulation were researched. The energy benefit gained by using cascade structures has been defined.
\end{abstract}

Keywords: Noise Resistant Encoding, Cascade Encoding, Turbo-Encoder, Encoding Energy Benefit

\section{Introduction}

Modern-day achievements in electronics provide possibility to realize quite complicated algorithms for digital processing signals in transmitters and receivers of the communication systems. This results in enhanced transmission quality in digital systems as compared with analog communication systems. Data receiving errors are inherent to functioning data transmission systems what's caused by hindrance and noise in communication channels. To reduce influence of errors in receiving symbols on receiving data blocks quality, the means of noise resistant encoding are applied to detect and correct single and group errors and resend the data block at request. Noteworthy is that encoding methods are performed with interleaving symbols, which is used for breaking error groups into uncorrelated single errors [1]. As shown in [1,2] all error protective methods are based on applying excessive symbols which are added to informative ones and related to them by special equations which enable detecting errors. Comparing codes is reasonable by the degree of their characteristics' approach to Shannon threshold [3]. Theoretically this threshold determines potentially achievable signal to noise ratio for fixed encoding rate. Increasing energy effectiveness due to using codes is characterized by the value of equivalent energy benefit [1]. To improve data processing effectiveness in data transmission systems with phase modulation requires solving the following problems: improving reliability of data transmission; defining structure of data transmission system; giving recommendations to type of noise resistant codes and methods for their encoding. Analyzing the current problem showed that optimizing problem of improving effectiveness in data transmission system can be performed by using systematic approach to changing features of the discrete signals in communication channel. The effectiveness of parameters can be changed in the wide range. Research will be conducted by using method which is based on using multi-position signals and correction codes, what is a combination of those. The research objectives include studying possibility of using methods for noise resistant encoding in data transmission channels which are based on so called turbo-codes and cascade codes with turbo-encoding. The paper is devoted to these objectives. 


\section{Creating Scheme of Digital Data Transmission Channel with Noise Resistance Encoding}

Simultaneous improving energy and spectrum effectiveness of data transmission is possible in case of applying multi-positional signals together with noise resistant encoding. The idea can be expressed as the following: the signals to be created are such which areas in multi-positional space are tightly packed (what provides frequency effectiveness) and at the same time are wide separated (what provides high energy effectiveness). The manipulation methods types BPSK, QPSK and convolution codes [2] are implemented in data transmission channels in particular in satellite telecommunication channels. Combining signals and codes, which comply with conditions given above, makes signal-code structures. Multi-positional signal therewith makes "inner code". The problem of building such signal-code structures (SCS) consists in combining contradictive features of "tight" multi-positional signals (high frequency effectiveness) and noise resistant codes (high energy effectiveness) in unified structure that provides simultaneous increasing as energy effectiveness $\beta$ so frequency one $\gamma$ in correspondence with (1) [4].

$$
\beta=\frac{1}{E_{b} / N_{0}}=\frac{N_{0}}{E_{b}}, \gamma=\frac{\log M}{N}
$$

where $M$ - quantity of code symbols used in forming a signal, $N$ - signal space dimensions, $N_{0}$ - spectrum density of noise power, $E_{b}$ - energy taken to transmit one data bit.

Transition to multi-positional signals allows increasing partial rate of data transmission. However this lowers noise resistance as growing positions lowers energy effectiveness. Effective codes should have long structure similar to structure of random noise, therewith growing code length causes growing complexity of decoding algorithm dramatically. Therefore the problem to solve is search and synthesis of codes with high correction level and at the same time acceptable decoder complexity.

At the present time the most noticeable achievement of noise resistant encoding theory is developing turbo-codes. Such codes are formed as result of composing two or more comprising codes which make variants of alternating one data sequence. Unlike convolution codes when decoder gives strait decoded symbols in cascade scheme [1], turbo-code uses soft decoding scheme providing acceptable operation of decoding algorithm. Thus, in case of using systems with turbo-encoding, decoding consists in transmitting soft scheme for making decision from output of one decoder to input of another one and repeating this procedure until the reliable decision. As shown in $[1,4]$ applying digital phase manipulation to data transmission systems is originally followed by insufficient noise resistance. The modern-days solution to this problem may be found in combining digital manipulation with grid encoding in particular by using trellis coded modulation. Chosen kind of phase modulation and noise resistant code forms signal-code structure (SCS).

The process of building turbo-codes (TC) will be next considered. Figure 1 demonstrates encoder TC which generally can be represented as parallel joining two recursive systematic encoders.

Both convolution encoders work at $1 / 2$ rates. Data is delivered to the input of second convolution encoder from output of pseudo-random interleaver [1]. Encoding rate of such turbo-encoder will make $1 / 3$ in general. Multiplexor performs perforation [2] to $1 / 2$ rate. Owing to using systematic convolution encoders the systematic and verifying parts can be selected. Thus, the data transmission channel gets two code blocks to transmit: first code block, which consists of mixed data part; and verifying part of the lower convolution encoder. The literature review allows making the following conclusions [1]: the interleaver structure is negligible to its effectiveness. While decoding the block can be divided into two code blocks, which will be identical (fig. 2). In this case two decoders can be used, each of them performs decoding of its own code block. The decoded data of the first (second) decoder with taking into account interleaving can be used as a priory data for the second (first) decoder in order to finally clarify the decoding result, so in this way forming feedback between decoders of two code blocks. Such operations can be multiply repeated (performing several iterations). For an instance figure 3 demonstrates dependence of bit error rate (BER) on signal to noise ratio for Gauss channel with turbo-encoding with various size of data block $L$ and various quantity of iterations. This is the way how turbo-decoding is performed. Every decoder here makes decisions regarding transmitted symbol on the base of maximal a posteriori probability [2].

During first iteration the estimations ("soft" decisions) of informative symbol from systematic and first verifying part of the code block are incoming to the input of first decoder. In the output of the first decoder the estimation ("soft" decision) of data symbol is formed, which will be furthermore used as a prior information about it for the second decoder. This decoder performs estimation of symbol in the output of interleaver on the base of verifying part of the second code word. During second and next iterations of decoding this estimation is renewed and used as a prior information regarding transmitted symbol for the first decoder. So inputs of both elementary decoders receive "soft" decisions. The decoding result in the decoder's output is also "soft" decision. The final decision on the transmitted informative symbol is made by the second decoder. Finalizing decoding process is performed either after fulfilling the specified quantity of iteration cycles $\mathrm{Q}$ or after the correction value of decoding result reaches the specified threshold. Asymptotic threshold for turbo-codes is defined by the expression: 


$$
P_{\text {error }} \geq \frac{1}{L} \cdot Q\left(\sqrt{2 d_{f} \cdot R \cdot \frac{E_{b}}{N_{0}}}\right) \cdot \sum_{\omega=1}^{L} \omega \cdot C_{\omega, d_{f}},
$$

where $L$ - the block size to be interleaved, $d_{f}$ - free distance of turbo-code [3], $\omega$ - code weight, $C_{\omega, d_{f}}$ - the quantity of code sequences with weight $d_{f}$, $R$ - encoding rate.

Figure 4 represents simulative scheme of data transmission system with turbo-encoding. The sequence formed by Bernoulli binary generator is delivered to turbo-encoder sub-system, which comprises two convolution encoders and interleaver, modulator of phase-manipulated signal, gauss channel AWGN, which is used as the communication channel in whose output the turbo-decoder sub-system (fig. 5) is placed, which in turn comprises two decoders, interleaver and de-interleaver according to scheme in figure 2.

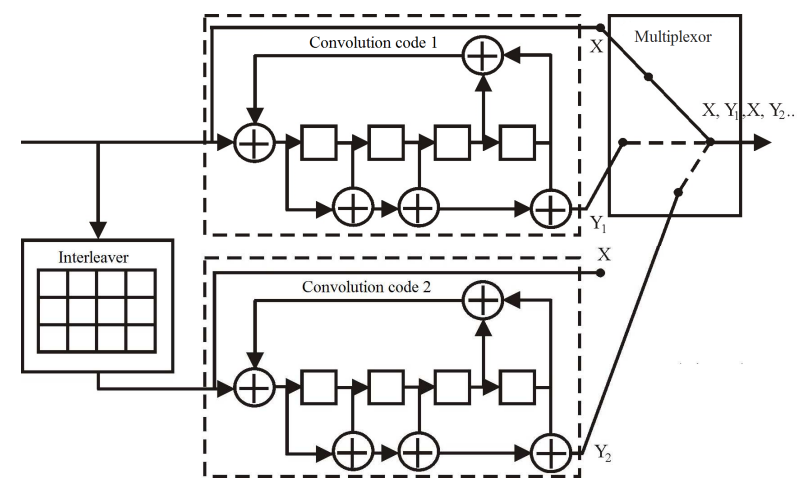

Figure 1. Turbo-encoder scheme.

The figure 6 shows code sequences in the output of
Bernoulli binary generator and in the output of turbo-code decoder obtained by means of Scope tool.

The method for highly effective code will be used in the way of combining two or more component codes to create the cascade encoding scheme. As indicated later such codes can correct errors in much longer codes and have structure which allows relatively simple decoding of medium complexity. Turbo-code is generated by parallel cascading two or more elementary component convolution codes divided by interleaver. Using systematic convolution codes, on other equal conditions, guarantees best characteristics for turbo-code. The necessary link of turbo-encoder is interleaver.

Due to interleaver the process of forming code combinations in turbo-code is nearly random. That's why turbo-code with large block size can be characterized as long random code, in accordance to Shannon theorem exactly such codes are needed for data transmission at the rate approaching to communication channel capacity. Turbo-code will be used to renew structure of

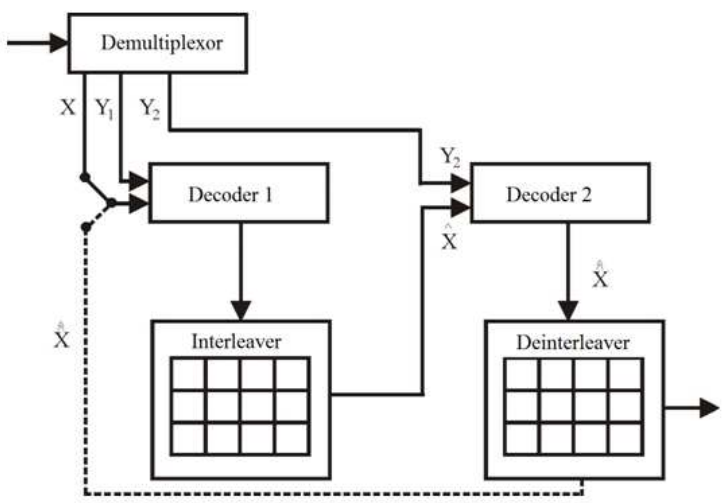

Figure 2. Turbo-code decoder scheme.

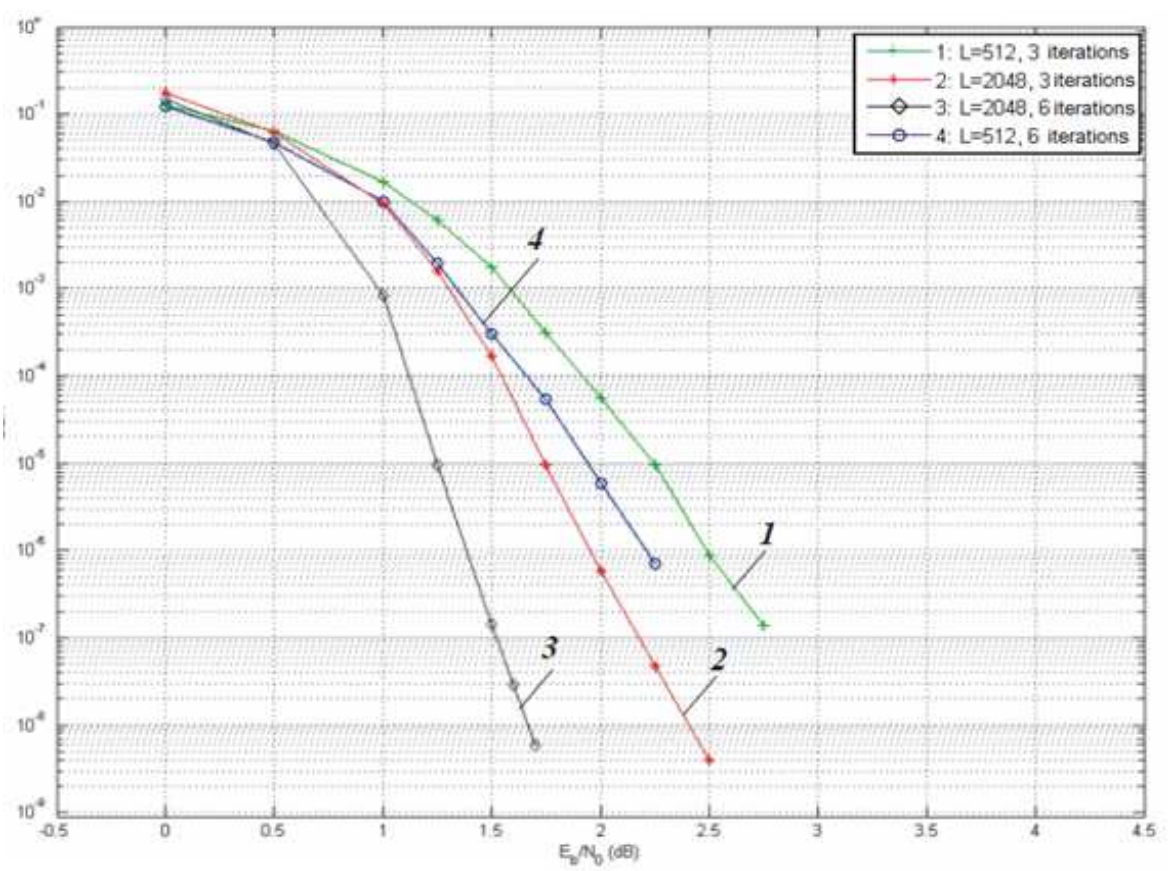

Figure 3. Dependences of bit error rate BER on signal to noise ratio ( $\left.E_{b} / N_{0}\right)$ for $A W G N$ channel. 
cascade encoding with iterative decoding algorithm for connected code sequence.

The turbo-encoding has specific features to consider. In convolution codes at decoder output the rigid decoded bits are formed. In cascade scheme there is no limitations for decision. For best using information obtained from decoder, decoding algorithm should apply soft decoding scheme. Here will be used a concept described as soft making decision scheme that is transmitting data from output of one decoder to input of another one repeating this procedure until reliable decision. The dependence of changing bit error on receiving symbols (Bit Error Ratio) on signal to noise ratio in communication channel for signal-code structure on the base of turbo-code TC having configuration $(37,21)$ was defined. Figure 7 also represents asymptotic limit for turbo-code calculated by formula (2)

Analysis of dependences shown in fig. 7 provides making following conclusions. At BER probability less $10^{-5}$ the TC characteristics slope lowers as $E_{b} / N_{0}$ value is growing, what indicates saturation effect of BER probability. To reach low error probability, for an instance about $10^{-8}$, obviously with the help of turbo-code, is possible at high values of $E_{b} / N_{0}$. Besides, comparing fig. 7 with fig. 8, represented in paper [1], testifies that at low BER probability TC will not be effective as compared with cascade codes (the curve 3 in fig. 7 and curve 5 in fig. 8 are compared at BER value $10^{-6}$ ).

Turbo-code researched above had the following parameters: symbols quantity in the encoder input $k=$ 65523, turbo-encoder structural length $K=5$, interleaver length $L=k+K-1=65536, R=1 / 2$, code word length $n=L / R=131072$.

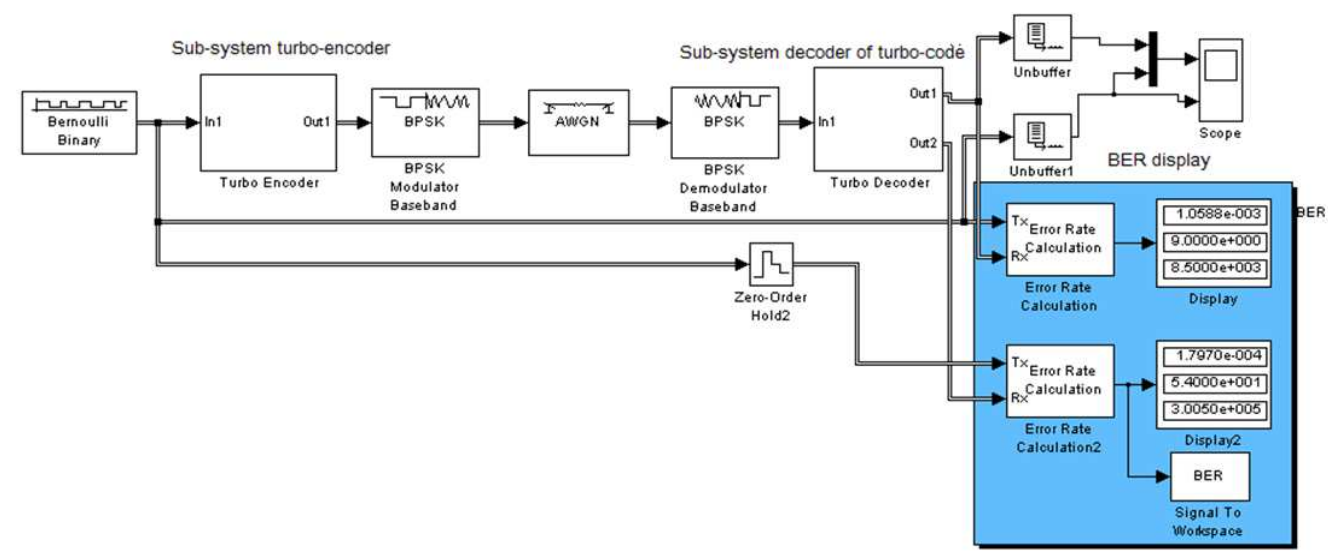

Figure 4. Simulation scheme of data transmission with phase manipulation and turbo-encoding

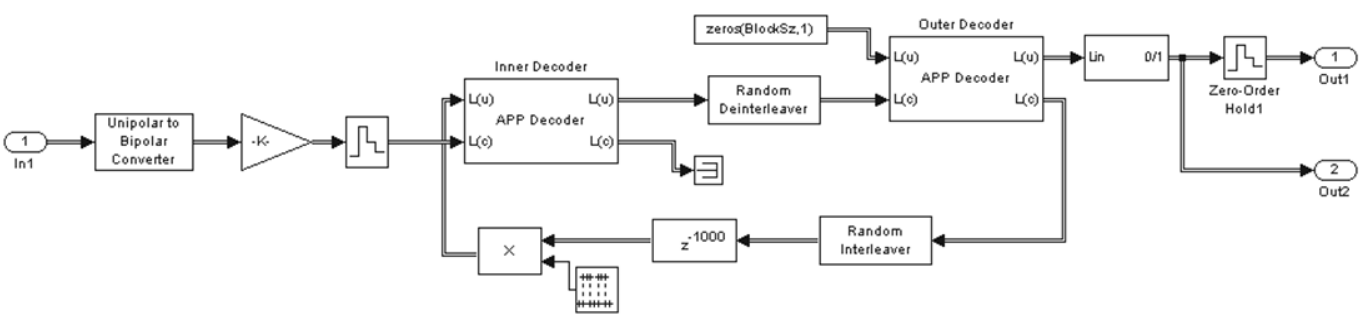

Figure 5. Simulation model of turbo-code decoder sub-system

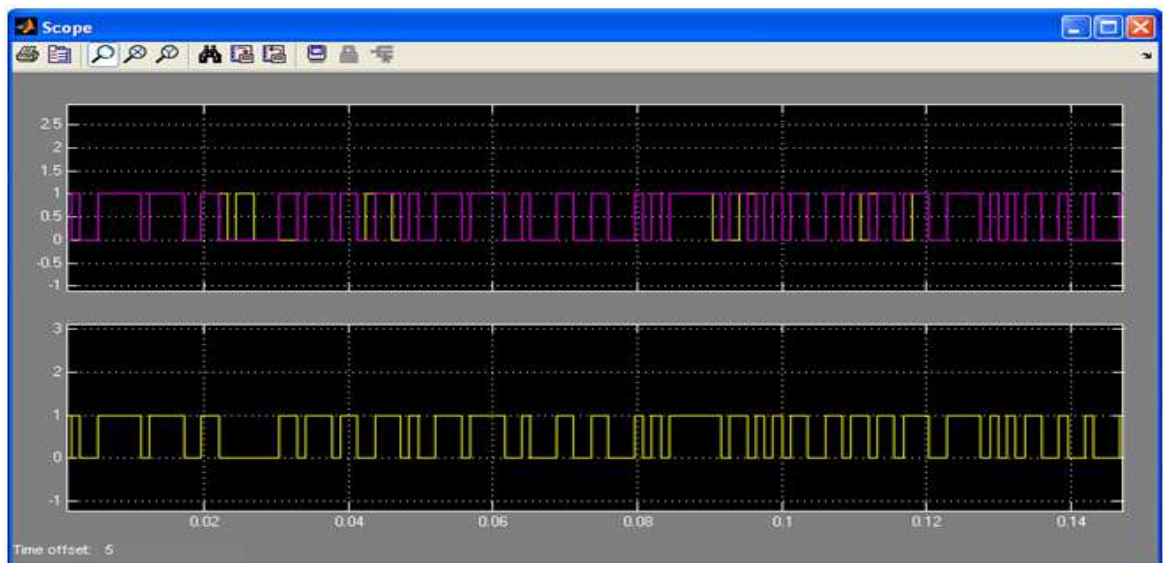

Figure 6. Code sequences in the output of Bernoulli binary generator and in the output of turbo-code decoder. 
Literature review allows making the following conclusions. Turbo-encoders of the studied structure at $Q-$ decoding iterations have the same complexity by the quantity of need operations, which are adding and multiplying operations, as decoder Vitebri [2] of convolution code. The computation complexity of turbo-encoder calculated over one data bit does not depend on data block length $k$. However noticeable is growing volume of decoder memory and decoding delay for TC when $k$ is growing.

The research of influence that various transport blocks and quantity of decoding iterations have on workability of the data transmission system with turbo-encoding will be conducted. Conducting experiments on various transport block lengths allows to observe various quantity of code blocks in transport block resulted from its segmenting while decoding process. To conduct the modeling the nominal time of $1 \mathrm{~ms}$ was specified for selecting transport module. Research results are represented in fig. 9 a, b. Figure 9 demonstrates result of decoding in telecommunication system of data transmission with turbo-encoding. In particular, six iteration cycles were selected while decoding process in fig. 9 a.

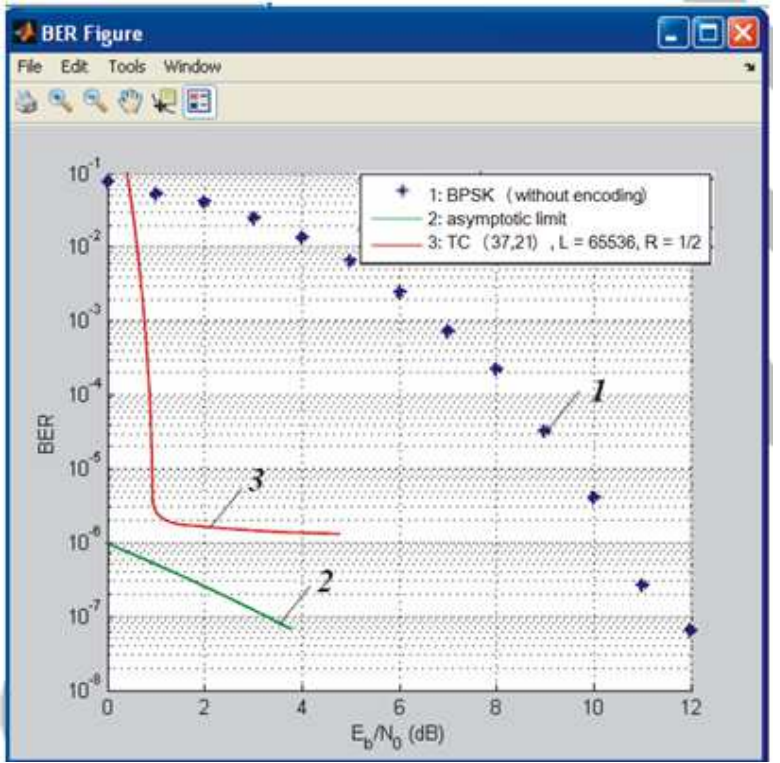

Figure 7. Dependences of bit error rate BER on signal to noise ratio $\left(E_{b} / N_{0}\right)$ for AWGN channel:1 - without encoding (BPSK); 2 asymptotic limit TC;3 - TC (37,21), $R=1 / 2$

The decoding result is obtained at maximal quantity of decoding iterations and noise level, for typical size of transport block 4584, 9144, 15264 and 24496 bits and verifying additive input of 24 bits [2]. The research was conducted with turbo-code of transport block size 75376 added by verifying input for detecting errors at receiving side fordecoded block. This resulted in transport block 75400. Blocks highlighted in the figure by blue color correspond to processed components of transport channel, and those to have other color represent decoding errors.
Figure $9 \mathrm{~b}$ represents assessment of decoding results at eight iterations given. Transport block is defined to have no errors. Adding two iteration cycles enabled to correct errors in five code blocks which had errors detected previously, whereas the other code blocks were decoded using the same quantity of iterations as well as in the first modeling.

\section{Building Scheme of Transmission System with Cascade Turbo-Encoding and Measuring Energy Benefit of Encoding}

The paper [1] considered possibilities of building schemes of data transmission using noise resistant encoding.

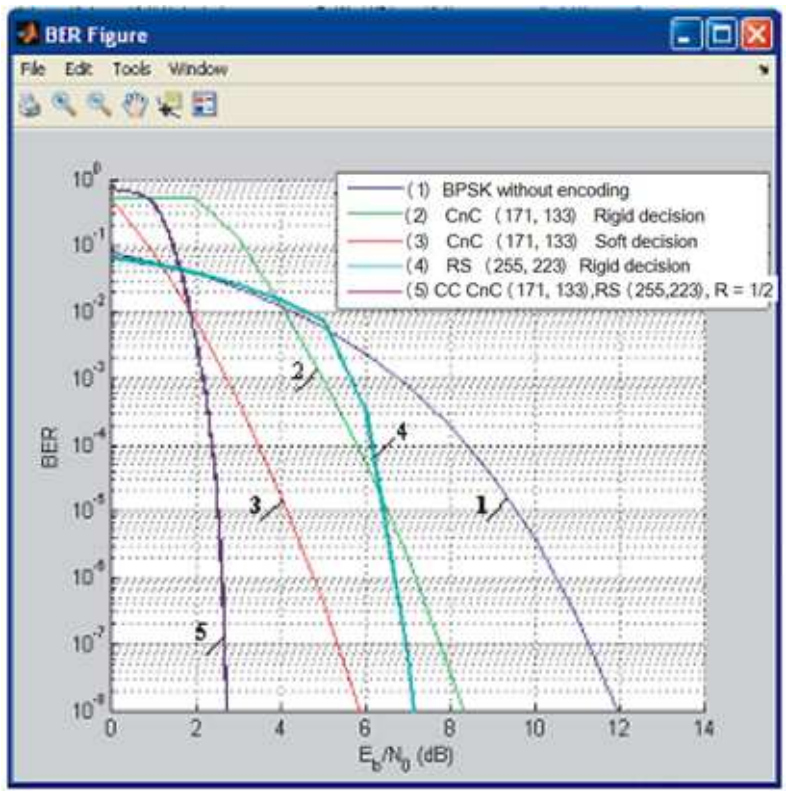

Figure 8. Dependences of bit error rate BER on signal to noise ratio ( $\left.E_{b} / N_{0}\right)$ for AWGN channel: 1 - without encoding (BPSK); 2 CnC(171,133) Riding decision; 3 - CnC(171,133) Soft decision; 4 $R S(255,223)$ Riding decision; 5 - CC: $C n C(171,133), R S(255,223), R=1 / 2$

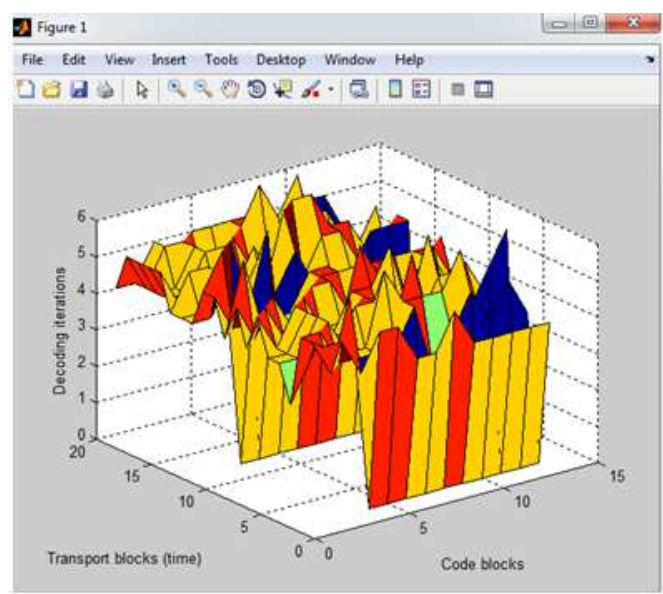




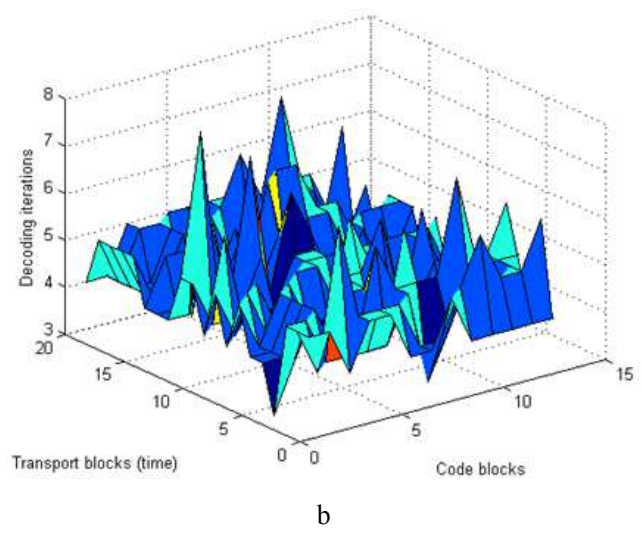

Figure 9. Results of decoding transport blocks of turbo-codes in six iteration cycles $-a$ and eight iteration cycles $-b$ while decoding.

In particular, using differential (relative) encoding in systems without noise resistant encoding entails multiplying errors. The relative encoding is reasonable to use in systems with noise resistant encoding. In this case the order to engage relative and noise resistant encoder does matter. The inner and outer relative encoding can be selected here. On the base of formulated ideas [1] concerning realization of the digital data transmission channel the following conclusions can be made. To realize codes with large block length and high correcting capability in data transmission channel the cascade codes are reasonable to use. In this case several encoding levels should be used. The scheme with two levels is the most reasonable. The Reed-Solomon code can be used as the outer one, variety of the others can be used as the inner code. The turbo-codes will be considered to use as inner codes and Reed-Solomon [1] code as the outer one.

In this case the characteristics of developed structure can be considered such which approaches to their potential for such combination of codes. The scheme of data transmission system with cascade encoding will be built and effectiveness of cascade code with inner TC (37.21) and outer Reed-Solomon code will be assessed. The RS $(255,223)$ code which was researched in [1] will be taken for example now; it is used in satellite telecommunication digital data transmission channels [4]. In this case the main scheme of data transmission system is represented in figure 10 .

Analyzing results obtained above for turbo-code (fig. 4) asymptotic limit of TC is noticed to be near experimental dependence only in the range of BER $<10^{-5}$. So using expression (2) is not appropriate for calculation of characteristics of cascade code with inner turbo-code. In this case the formula for Reed-Solomon decoder given in [1] should be used to find bit error probability of binary symbols:

$$
P_{b} \leq \frac{2^{m-1}}{2^{m}-1} \sum_{i=t+1}^{n} \frac{i+1}{n} \cdot C_{n}^{i} \cdot P_{\text {sim }}\left(1-P_{\text {sim }}\right)^{n-i},
$$

where $P_{\text {sim }}=1-\left(1-P_{c}\right)^{m}$ - error probability $2^{m}-$ th symbol, $P_{c}$ - bit error probability in the channel.

Reed-Solomon codes are block codes $(n, k)$, which have maximal distance $d=n-k+1$. For these no binary codes which are defined over Galua field $G F(q)$, the block length makes $n=q$-1 (normally $q=2^{m}$, then code corrects $2^{m}-$ th symbol). Therewith correcting $t$ errors requires $2 t$ correcting symbols. Every symbol of Reed-Solomon code word consists from a number of bits. Decoding can correct $t=(n-k) / 2$ symbols, which are defined as having errors. All operations on encoding and decoding procedures are conducted in Galua field $G F\left(2^{x}\right)$ (final product which consists from $2^{x}$ components) by polynomial module or by bytes structure in field $G F\left(2^{8}\right)$.

Figure 11 demonstrates dependences of error probability BER on signal to noise ratio $\left(E_{b} / N_{0}\right)$ for cascade code described above, and also for convolution code $(\mathrm{CnC})$ (CnC $(171,133), R=1 / 2)$ in case of decoding by "soft" Vitebri algorithm, BPSK - manipulated signal without encoding and turbo-code. Besides, assuming found dependence of error probability BER on signal to noise ratio $\left(E_{b} / N_{0}\right)$ for cascade code (fig. 8, curve 5) makes conclusion that cascade code (curve 4 in fig. 11) removes satura- tion effect inherent for TC.

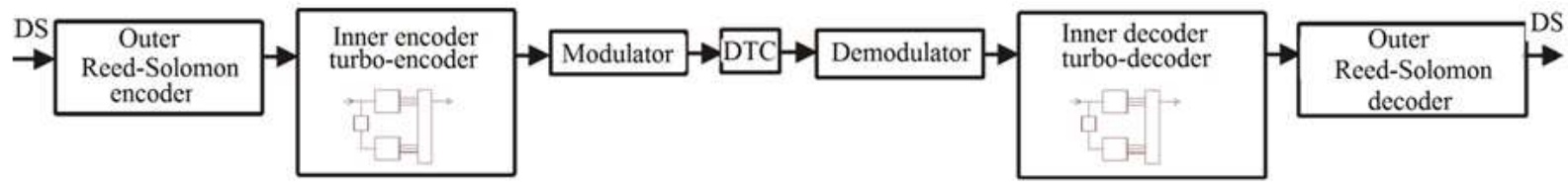

Figure 10. Structural scheme of data transmission channel with cascade encoding: DS - data stream; DTC - data transmission channel.

Next compared are two curves: curve 5 (fig. 8) for dependence of error probability BER on signal to noise ratio $\left(E_{b} / N_{0}\right)$ for cascade code formed having the following structure: outer Reed-Solomon encoder; interleaver and inner convolution encoder and curve 4 (fig. 11), which represents dependence of error probability BER on signal to noise ratio $\left(E_{b} / N_{0}\right)$ for cascade code formed as the structure shown in fig. 10: outer Reed-Solomon encoder, inner - turbo-encoder. The conclusion is made that benefit of cascade code structure with turbo-encoder in this case makes about $1.8 \mathrm{~dB}$ for level BER $10^{-6}$. The noteworthy is that saturation effect in modern micro-schemes is removed by including additional encoding level [3]. For the purpose of finding energy benefit of cascade encoding the simulation modeling was conducted.

Simulation model of data transmission channel is built up 
to the next structure: cascade encoding (outer Reed-Solomon encoder $(255,247)$, inner encoder turbo-encoder $(L=1554, \quad R=1 / 2$, QPSK)). Modeling assumed that frequencies of quadrature generators in receiving and transmitting sides are phase matched. Modeling scheme of data transmission channel was aimed at finding encoding energy benefit (EEB) providing changing encoding rate of turbo-encoder for the specified code ( $R=1 / 2$ (curve 3 in fig. 12) and $R=1 / 3$ (curve 4 in fig. 12). In particular, figure 12 represents calculation results of bit error dependence BER for given above signal-code structures (SCS) on signal to noise ratio $\left(E_{b} / N_{0}\right)$ for AWGN channel at various rates. Figure 12 represents the curve of BER changes on signal to noise ratio $\left(E_{b} / N_{0}\right)$ for AWGN for unencoded QPSK (curve 1) and also curve (curve 2 ) obtained by simulative modeling of data transmission channel with cascade encoding of SCS type: outer encoder - RS (255, 247), interleaver, inner encoder - CK $(171,133)$, QPSK, $R=1 / 2$.

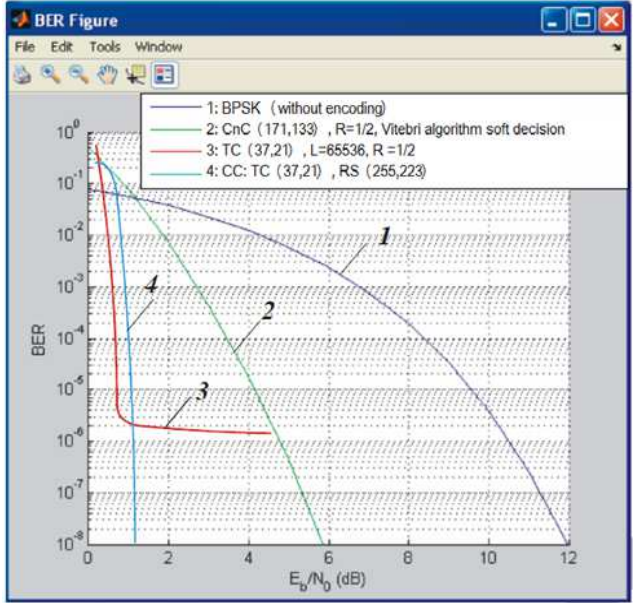

Figure 11. Dependence of bit error BER on signal to noise ratio $\left(E_{b} / N_{0}\right)$ for AWGN channel: 1 - without encoding (BPSK); 2 - CnC (171,133); $3-T C$ (37,21), $R=1 / 2 ; C C: T C(37,21), R S(255,223)$

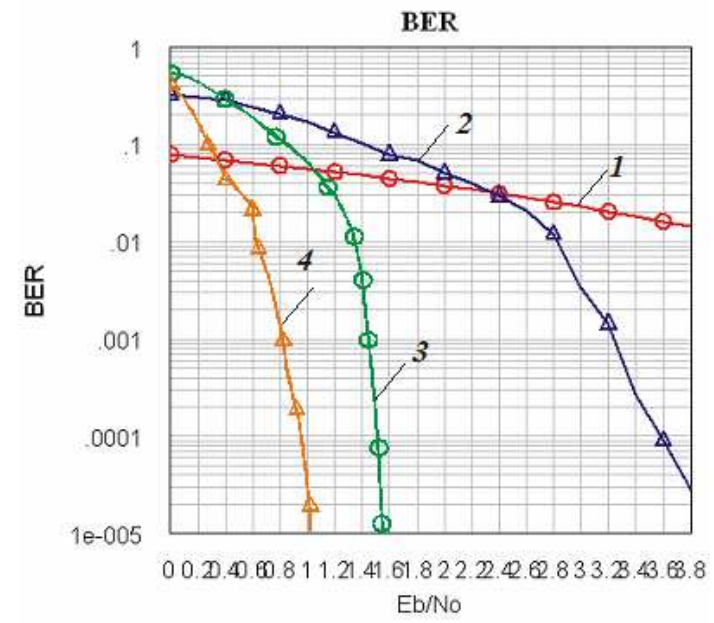

Figure 12. Dependence of bit error BER on signal to noise ratio ( $E_{b} / N_{0}$ ) for AWGN channel with cascade turbo-encoding: 1 - without encoding (QPSK); $2-C C: R S(255,247)$, interleaver, $C n C(171,133)$, QPSK, $R=1 / 2 ; 3-T C: R=1 / 2,4-T C: R=1 / 3$
Provided that encoding rate is $1 / 2$ for the specified code, the energy encoding benefit made $2.3 \mathrm{~dB}$ for bit error probability $10^{-5}\left(\mathrm{BER}=10^{-5}\right)$ as compared with cascade code used in data transmission channel and having structure: outer Reed-Solomon encoder of type $(255,247)$, interleaver, inner code - convolution $(\mathrm{CnC})$ of type $(171,133)$ and quadrature phase modulation - QPSK. In case of realizing data transmission channel at encoding rate $1 / 3$, EEB equaled $2.8 \mathrm{~dB}\left(\mathrm{BER}=10^{-5}\right)$, as compared with cascade code having the following structure: SCS RS $(255,247)$, interleaver, CnC (171, 133), QPSK, $R=1 / 2$. Changing code structure, encoding rate in case of turbo-encoding leads to increasing EEB. As compared with unencoded phase manipulation (curve 1 in fig. 12 ) $\mathrm{EEB} \approx 8.5 \mathrm{~dB}$ for $R=1 / 3$ and $7.9 \mathrm{~dB}$ for $R=1 / 2 \quad\left(\mathrm{BER}=10^{-5}\right)$. Selection of data transmission channel structure should be done by applying cascade codes and cascade turbo-codes optimal to correct errors, provide excessive and fast encoding.

\section{Conclusions}

The paper make the following statements: turbo-codes are formed as result of composing two or more component codes, which are variants altering one data sequence; and decoding procedure consists in transmitting soft scheme of decision making from output of one decoder to input of another one and repeating this procedure until the reliable decision. It has been defined that enhancing noise resistance in digital telecommunication channels is achieved by using schemes of cascade turbo-encoding. The slope of TC characteristics is defined to lower at growing value of $E_{b} / N_{0}$, what testifies of saturation effect in error probability. From the research it has been found that encoding energy benefit (EEB) for cascade code is formed by the structure: outer Reed-Solomon encoder, inner turbo-encoder makes about $1.8 \mathrm{~dB}$ for level BER $10^{-6}$ as compared with cascade code CC: RS $(255,223), \mathrm{CnC}$ (171, $133)$, BPSK, $R=1 / 2$. The structural scheme of data transmission channel with cascade turbo-encoding has been developed. It is defined that in case of encoding rate $1 / 2$ $\mathrm{EEB} \approx 2.3 \mathrm{~dB}\left(\mathrm{BER}=10^{-5}\right)$ as compared with cascade code having the following structure: SCS RS (255, 247), interleaver, $\mathrm{CnC}(171,133)$, QPSK, $R=1 / 2$. In case of realizing data transmission channel at encoding rate $1 / 3$ EEB equaled $2.8 \mathrm{~dB}\left(\mathrm{BER}=10^{-5}\right)$, as compared with cascade code having structure: SCS RS (255, 247), interleaver, $\mathrm{CnC}(171,133)$, QPSK, $R=1 / 2$. Changing code structure, encoding rate in case of turbo-encoding leads to increasing EEB. It has been developed Simulink simulation model for data transmission with cascade turbo-encoding with the purpose to research encoding effectiveness for protection from errors in digital telecommunication channels. 


\section{References}

[1] J.M. Boiko, A.I. Eromenko. (2014). Solutions Improve Signal Processing In Digital Satellite Communication Channels. 20th International IEEE conference on microwaves, radar and wireless communications. MIKON-2014. June 16-18, pp. 126-129, Gdansk - Poland.

[2] Juliy Boiko, Oleksander Eromenko. (2014). Noise immunity assessment in telecommunication systems with cascade encoding structures. TCSET'2014 IEEE. 25 February-1 March, pp. 431-433, Lviv - Slavske.
[3] Boiko J.M. (2013). Improving effectiveness for processing signals in data transmission channels with phase manipulation. 23rd International IEEE Crimean Conference "Microwave \& Telecommunication Technology" September 9-13, pp. 262-263, 2013, Sevastopol.

[4] Juliy Boiko, Victor Stetsiuk, Victor Michan. (2012). Improving noise immunity of QPSK demodulation of signals in digital satellite communication systems. TCSET'2012 IEEE. 21 - 24 February, pp. 257, Lviv - Slavske.

[5] Boiko J.M. (2013). Modeling satellite data transmission channel with cascade signal-code structures. Modern directions of theoretical and applied researches 2013 SWorld Web Of Science, Scopus, 19-30 March, Odessa. 